\title{
Prion Protein is a Novel Modulator of Influenza: Potential Implications for Anti-Influenza Therapeutics
}

\section{Suehiro Sakaguchi* and Junji Chida}

Division of Molecular Neurobiology, Institute for Enzyme Research (KOSOKEN), Tokushima University, 3-18-15 Kuramoto, Tokushima 770-8503, Japan

*sakaguchi@tokushima-u.ac.jp

DOI: https://dx.doi.org/10.21775/cimb.037.021

\begin{abstract}
Worldwide spread of influenza A virus (IAV) strains, which are resistant to currently available antiinfluenza agents such as viral neuraminidase inhibitors, has encouraged identification of new target molecules for anti-influenza agents. Reactive oxygen species (ROS) causing oxidative stress play a pivotal role in the pathogenesis of lung injuries induced by infection with IAVs, therefore suggesting that anti-oxidative therapeutics targeting cellular molecules could be beneficial against IAV infection without inducing drug-resistant IAV strains. We recently found that the normal cellular prion protein, $\mathrm{PrPC}$, whose conformational conversion into the amyloidogenic isoform, PrPSc, in the brain is a key pathogenic event in prion diseases, is expressed by lung epithelial cells and exerts a protective role against IAV infection in mice by reducing ROS in infected lungs. The $\mathrm{Cu}$ content and activity of antioxidative enzyme $\mathrm{Cu} / \mathrm{Zn}$-superoxide dismutase, or SOD1, were lower in the lungs of $\mathrm{PrP}^{\mathrm{C}}$-knockout mice, suggesting that the anti-oxidative activity of $\mathrm{PrPC}$ is probably attributable to its function of activating SOD1 through regulating $\mathrm{Cu}$ content in lungs. Here, we introduce $\mathrm{PrPC}$ as a novel modulator of influenza and its potential implication for anti-oxidative therapies for IAV infection. We also introduce other candidate targets reported for antioxidative anti-influenza therapies.
\end{abstract}

\section{Introduction}

Influenza A viruses (IAVs) are enveloped, negative sense, single-stranded RNA viruses, causing seasonal epidemics of influenza affecting about $20 \%$ of the world population annually, with 250,000-500,000 deaths each year (Fiore et al., 2008). The young and elderly and those with underlying chronic diseases in lung or cardiovascular systems are particularly vulnerable to IAV infection (Fiore et al., 2008). There have been so far four pandemics of influenza in the past; the 1918-1919 Spanish flu pandemic caused by infection with IAV subtype H1N1, the 1957-1958 Asian flu pandemic by H2N2, the 1968-1970 Hong Kong flu pandemic by H3N2, and the 2009-2010 swine flu pandemic by H1N1, causing greater than 50 million deaths worldwide (Nickol and Kindrachuk, 2019). Potential risks of emergence of new IAV subtypes and their worldwide spread leading to new flu pandemics are still high. In addition, many sporadic infections with highly pathogenic avian influenza virus (HPAIV) subtype H5N1 to humans, causing high morbidity and mortality in infected people, have been reported (Nunez and Ross, 2019). As of July 2018 , a total of 860 cases of H5N1 infection in humans have been confirmed and 454 deaths have been reported (Nunez and Ross, 2019). There have been only a few cases of humanto-human transmission of HPAIVs (Nunez and Ross, 2019), suggesting a very low likelihood of pandemic threat of H5N1 infection in human populations. However, there are potential risks that HPAIVs might change their pathogenic properties in humans and produce new types of progeny viruses, which are more susceptible to humans and more easily spread among human populations through human-to-human infection, eventually causing new flu worldwide in the world. 
Vaccines against the viral surface glycoprotein hemagglutinin (HA) are currently available as a prophylactic measure against IAV infection (Yamayoshi and Kawaoka, 2019). However, they are only effective if they target the current circulating viruses. Furthermore, they also cannot reduce the ongoing threat of new pandemic strains since production of the vaccines takes a long time. Inhibitors of the viral neuraminidase (NA), which is essential for release of IAV progenies from infected cells, are the currently available major antiviral agents (Robson et al., 2019). The efficacy of the agents has been clearly demonstrated. However, the agents need to be used within 48 hours of onset and are most effective within 24 hours (Robson et al., 2019). In addition, certain strains of IAVs have already acquired resistance to these anti-influenza agents and have been spreading in human populations worldwide (Robson et al., 2019). The anti-influenza agents target the viral molecules, thereby stimulating the emergence of drug-resistant IAVs, which carry mutations in the genes encoding those molecules. Therefore, identification of new target molecules for anti-influenza agents is urgently awaited.

Many cellular molecules play a crucial role in the pathogenesis of IAV infection (Marques et al., 2019), suggesting that these cellular molecules could be plausible targets for new anti-influenza agents. Lines of evidence indicate that oxidative stress plays a pivotal role in the pathogenesis of IAV infection (Akaike et al., 1990; Oda et al., 1989; Vlahos and Selemidis, 2014). We recently identified that the normal cellular prion protein, $\operatorname{PrPC}^{\mathrm{C}}$, could have a protective role against lethal infection with IAVs in mice by functioning as an anti-oxidative molecule by regulating an anti-oxidative enzyme in lungs (Chida et al., 2018). In this review, we introduce $\mathrm{PrPC}^{\mathrm{C}}$ as a novel potential candidate target for anti-oxidative therapeutics against IAV infection, as well as other candidate targets reported for antioxidative anti-influenza therapeutics.

\section{Brief overview of IAV infection}

IAV infection begins with attachment of the virus to the surface of the airway or lung epithelial cells and alveolar macrophages through specific binding of the viral surface protein HA to either $\alpha 2,3-$ or $\alpha 2,6$ linked sialic acids expressed on the cell surface (Chutinimitkul et al., 2010; van Riel et al., 2010). Seasonal and pandemic IAV strains use $\alpha 2,6$-linked sialic acids for binding to the host cells while HPAIV strains use a2,3-linked sialic acids (Shinya et al., 2006; van Riel et al., 2010). a2,6-linked sialic acids are prominently expressed in the trachea, whereas a2,3-linked sialic acids are expressed by alveolar type II (ATII) epithelial cells (Shinya et al., 2006; van Riel et al., 2010). This specific expression of $\alpha 2,3-$ linked sialic acids in ATII cells, which is located in the respiratory tract terminus, alveolus, have been suggested to explain why HPAIV strains have difficulty infecting humans. Binding of IAVs to the cells triggers endocytosis of the virus into them. The acidic environment of the endosomes causes fusion of the virus with the endosomal membrane, resulting in release of the viral genome into the cells eventually leading to propagation of the viral progenies (Pinto and Lamb, 2006). The M2 viral protein is activated in the endosomes and forms an ion channel on the viral membrane, allowing entrance of protons into the viral core to dissociate the ribonucleoprotein complex (Pinto and Lamb, 2006). The newly replicated viral progenies are still bound to the host cells through linkage of HA of the progenies to sialic acids on the cell surface (Rossman and Lamb, 2011). The viral protein NA cleaves the link, allowing release of the progeny viruses from the host cells and spread of IAV infection in the lungs (Rossman and Lamb, 2011).

IAV infection can be cleared by the host immune systems. The innate immune system detects IAV infection through recognition of the virus by host cells via interaction of pathogen-associated molecular patterns, which are present in the virus or generated during infection, with cellular pattern recognition receptors such as the Toll-like receptors (TLRs), retinoic acid-inducible gene I (RIG-I), and NOD-, LRR- and pyrin domain-containing 3 (NLRP3) (Iwasaki and Pillai, 2014). TLR3 and TLR7 recognizes dsRNA and ssRNA in endosomes, respectively, and RIG-I detects 5 '-triphosphate viral ssRNA that is generated in the cytoplasm after IAV infection (Iwasaki and Pillai, 2014). These interactions between viral and cellular molecules trigger activation of innate immunity against IAV infection, leading to production of pro-inflammatory cytokines, recruiting immune cells into the infection sites, activating adaptive immune responses against IAV infection, eventually eliminating IAV infection (Iwasaki and Pillai, 2014). NLRP3 is expressed in monocytes, dendritic cells, neutrophils, macrophages, and epithelial cells and forms the multiprotein complexes called inflammasomes together with the ASC protein and pro-caspase 1 (Guarda et al., 2011). IAV infection triggers formation and activation of the NLRP3 inflammasomes, resulting in release of interleukin-1 $\beta \quad(\mathrm{IL}-1 \beta)$ and IL-18 and thereby 
consequently activating immune responses against IAV infection (Thomas et al., 2009). However, inordinate activation of these inflammatory responses against IAV infection causes excessive production of pro-inflammatory cytokines, the socalled 'cytokine storm', which is considered to be the underlying ccause for lethal infection by H5N1 viruses and the 1918 pandemic strain (La Gruta et al., 2007; Tumpey et al., 2005). It is also indicated that, although reactive oxygen species (ROS), which is generated during IAV infection, plays a protective role against IAV infection by oxidizing viral molecules, excessive production of ROS has a detrimental role against host cells or tissues during IAV infection (Akaike et al., 1990; Oda et al., 1989; Vlahos and Selemidis, 2014).

\section{Oxidative stress and cellular redox enzymes}

ROS are produced through enzymatic and nonenzymatic mechanisms after IAV infection and cause oxidative stress in infected cells (Vlahos and Selemidis, 2014). ROS are chemically reactive molecules containing oxygen, including superoxide, hydrogen peroxide, and hydroxyl radical, being steadily generated during normal cellular metabolism such as mitochondrial metabolism (Vlahos and Selemidis, 2014). However, excessive ROS overly oxidize proteins, lipids, and DNA, thereby damaging these molecules causing tissue damage, inflammation and apoptosis, eventually contributing to various pathological conditions (Akaike, 2001; Vlahos and Selemidis, 2014). Nicotinamide adenine dinucleotide phosphate (NADPH) oxidase, ubiquinone-cytochrome bc1, cyclooxygenases, lipoxygenases, cytochrome P450 reductases, and xanthine oxidase (XO) are major ROS-producing enzymes (Vlahos and Selemidis, 2014). Cells are also equipped with enzymatic and non-enzymatic anti-oxidative mechanisms to balance cellular redox homeostasis (Vlahos and Selemidis, 2014).

In general, ROS production begins with one electron reduction of molecular oxygen, resulting in generation of superoxide (Vlahos and Selemidis, 2014) (Figure 1). Superoxide is a negatively charged molecule, therefore being uncapable of crossing cellular membrane and executing its oxidizing activity in cellular compartments such as the phagosomes of neutrophils and macrophages to kill invading pathogens (Vlahos and Selemidis, 2014). Superoxide converts to hydrogen peroxide (Figure 1), which is uncharged and therefore free to diffuse across cell membranes leading to damage of neighboring cells and tissues (Vlahos and
Selemidis, 2014). The highly reactive hydroxyl radical is generated by the reaction between superoxide and hydrogen peroxide via the HaberWeiss reaction (Vlahos and Selemidis, 2014) (Figure 1). This reaction can be enhanced in the presence of free transitional metal ions (Vlahos and Selemidis, 2014). The Fenton reaction uses irons to produce hydroxyl radicals (Vlahos and Selemidis, 2014) (Figure 1).

NADPH oxidases are a family of multi-protein complex enzymes, consisting of flavin adenine dinucleotide and feme-containing Nox1, Nox2, Nox3, Nox4, and Nox5 as a catalytic subunit and up to five other molecules as regulatory subunits (Vlahos and Selemidis, 2014). p22 ${ }^{\text {phox }}$, DuoxA1 and DuoxA2 function to stabilize and express the enzymes in biological membranes, p6 $67^{\text {phox }}$ and Noxa1 activate the enzymes, and p47 phox, Noxo1 and $\mathrm{p} 40^{\text {phox }}$ function as organizers of the enzymes (Vlahos and Selemidis, 2014). Small GTPases Rac1 and 2 are also required for the enzyme function (Vlahos and Selemidis, 2014). Lung airway and alveolar epithelial cells express Nox1 and Nox2 (Carnesecchi et al., 2009; Takemura et al., 2010). Nox2 is also expressed in macrophages and neutrophils (Soucy-Faulkner et al., 2010).

Nitric oxide synthases (NOSs) generate nitric oxide (NO) through conversion of L-arginine to L-citrulline in the presence of oxygen (Akaike, 2001) (Figure 1). NO reacts with superoxide to produce highly reactive species peroxynitrite (Akaike, 2001) (Figure 1). There are three isoforms of NOS; epithelial NOS (eNOS), neuronal NOS (nNOS), and inducible NOS (iNOS) (Akaike, 2001; Antosova et al., 2017). iNOS is detected in various cell-types in lung tissues, including macrophages, the epithelium of the proximal and terminal bronchioles, and ATII epithelial cells (Pechkovsky et al., 2002; Warner et al., 1995). Pro-inflammatory cytokines enhance expression of iNOS in lungs (Ricciardolo et al., 2004).

Superoxide dismutase (SOD), catalase, and glutathione peroxidase are major anti-oxidative enzymes (Kinnula and Crapo, 2003; Sgarbanti et al., 2014). SOD catalyzes dismutation of superoxide into hydrogen peroxide (Kinnula and Crapo, 2003) (Figure 1). Hydrogen peroxides are then catalyzed into oxygens and water by catalases (Kinnula and Crapo, 2003) (Figure 1). There are three different SODs; intracellular copper/zinc-dependent SOD (Cu/Zn-SOD or SOD1), mitochondrial manganesedependent SOD (Mn-SOD or SOD2) and 
extracellular SOD (EC-SOD or SOD3) (Kinnula and Crapo, 2003). ATII epithelial cells express SOD1, SOD2, and catalase and are relatively resistant to oxidative stress (Kinnula et al., 1995). In contrast, ATI epithelial cells have low expression of antioxidative enzymes and are sensitive to enhanced oxidative stress (Kinnula and Crapo, 2003). SOD3 is expressed by bronchial epithelium, alveolar epithelial cells, and alveolar macrophages (Folz et al., 1997; Su et al., 1997). Alveolar macrophages also express catalase (Pietarinen-Runtti et al., 2000).

\section{Cellular redox enzymes are potential targets for anti-influenza therapeutics}

Given that excessive ROS are involved in the pathogenesis of IAV infection, reduction of ROS through either interfering with the oxidative mechanisms or enhancing the anti-oxidative mechanisms, or both, can be therapeutically beneficial for IAV infections. SOD1 is a major antioxidative enzyme in lungs, therefore suggesting that exogenously administered SOD1 could exert therapeutic effects against IAV infection (Oda et al., 1989). However, SOD1 is rapidly cleared from plasma with a halftime less than $5 \mathrm{~min}$ when intravenously administered (Oda et al., 1989). Oda et al. succeeded in prolonging the halftime of SOD1 markedly by conjugating pyran polymer to SOD1, and intravenously injected the pyran polymerconjugated SOD1 from days 5 to 8 after infection with IAV/Kumamoto/Y5/67(H2N2) (referred to as IAV/Kumamoto) (Oda et al., 1989). In marked contrast to native SOD1, pyran polymer-conjugated SOD1 successfully reduced the mortality of infected mice (Oda et al., 1989). The conjugates did not affect clearance of the virus from the lung (Oda et al., 1989). It was subsequently reported that SOD2 conjugated with pyran polymer was also effective against infection with IAV/WSN/33 (H1N1) (IAV/ WSN) in mice (Sidwell et al., 1996). Furthermore, it was shown that transgenic overexpression of SOD3 under the ATII cell-specific surfactant protein-C promoter was also effective against non-lethal infection with Hong Kong IAV/68 (H3N2), reducing inflammatory cytokines, oxidative stress and pathological changes in the lungs (Folz et al., 1999; Suliman et al., 2001). These results suggest that superoxide could be a major oxidant causing oxidative stress in lungs after infection with IAVs, and that treatments to mitigate superoxide in lungs could be therapeutic against IAV infection.

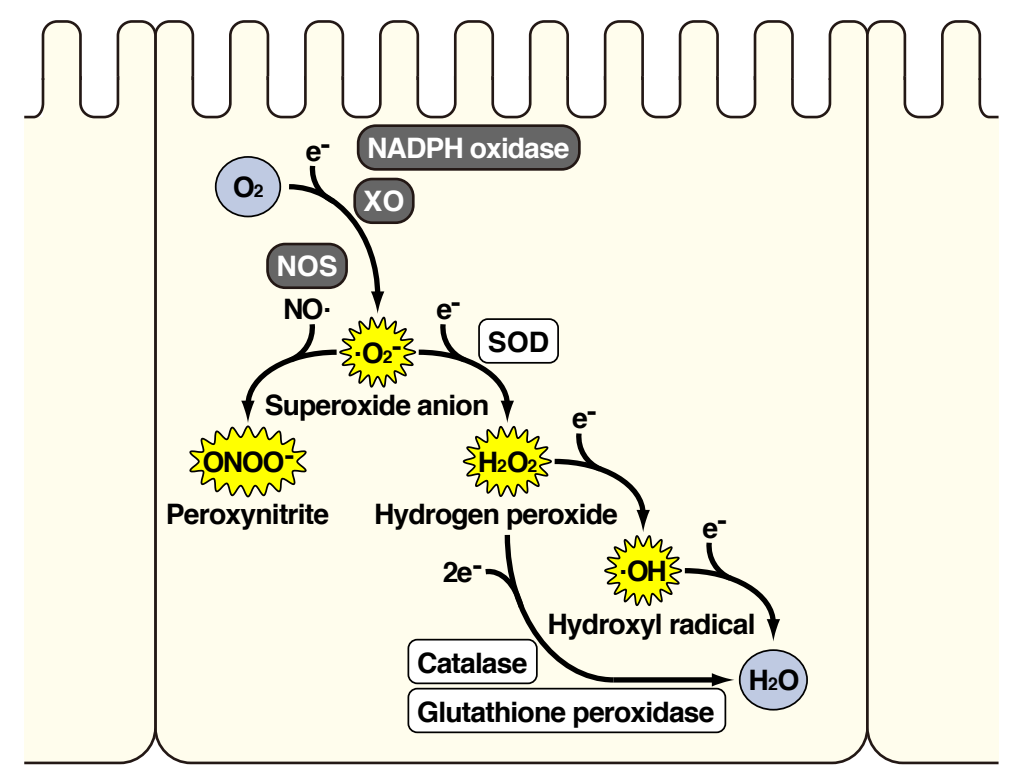

Figure 1. Schematic diagram of generation of ROS including superoxide anion, hydrogen peroxides, hydroxyl radicals, and peroxynitrite. Molecular oxygen is reduced to superoxide anions by NADPH, XO, and other oxidases, to hydrogen peroxides by SOD, then to hydroxyl radicals through the HaberWeiss reaction or the Fenton reaction. Hydrogen peroxides are catalyzed into water by catalases and glutathione peroxidase. NOSs generate NO, which reacts with superoxide to produce peroxynitrite. 
NADPH oxidases are also involved in the pathogenesis of IAV infection (Vlahos and Selemidis, 2014). However, different isoforms of the oxidase execute different effects on IAV infection. Mice deficient for Nox2, a subunit of NADPH oxidase predominantly detected in macrophages, have shown mild lung injuries after infection with IAV/X-31 (H3N2) and IAV/Puerto Rico/8/34 (H1N1) (hereafter referred to as IAV/PR8), with lower inflammatory responses and lower virus production in the lungs, compared to wild-type (WT) mice (Snelgrove et al., 2006; Vlahos et al., 2011). Moreover, daily intraperitoneal injection of the Nox2 selective inhibitor, apomycin, for 3 days prior to and for 3 days after infection with IAV/X-31 and IAV/PR8 was shown to reduce lung inflammation, with a decrease in number of inflammatory cells in bronchoalveolar lavage fluid and production of superoxide (Vlahos et al., 2011). Imai et al. showed that macrophages are crucial for causing acute lung injury after infection with H5N1 virus by producing Nox2 oxidase-dependent superoxide and oxidized phospholipids (Imai et al., 2008). These results suggest that Nox2 oxidase-mediated production of superoxide in macrophages could have a detrimental role in the pathogenesis of IAV infection, and that Nox2 oxidase could be a potential cellular target for anti-influenza agents. In contrast, Nox1 oxidase has been shown to be protective against IAV infection (Hofstetter et al., 2016; Selemidis et al., 2013). Mice deficient for Nox1 oxidase showed higher survival rate with lower morbidity after infection with IAV/PR8 (Hofstetter et al., 2016; Selemidis et al., 2013). T cells in the deficient mice infected with IAV/PR8 strongly responded against IAV/PR8 infection, leading to an increase of virusspecific $\mathrm{CD}^{+} \mathrm{T}$ cells in lungs and draining lymph nodes and cytokine-producing $T$ cells in lungs and spleens (Hofstetter et al., 2016). However, lung pathologies of Nox1 oxidase-deficient mice infected with IAVs seemed dependent on virus strains used (Hofstetter et al., 2016; Selemidis et al., 2013). No obvious differences in lung inflammation were reported in Nox1 oxidase-deficient mice infected with IAV/PR8, compared to control WT mice (Hofstetter et al., 2016). In contrast, inflammatory cells were highly infiltrated in the lungs of Nox1 oxidase-deficient mice after infection with IAV/ HkX-31 (H3N2), compared to control WT mice (Selemidis et al., 2013). The different effects of Nox1 and Nox2 oxidases on IAV infection could be attributable to their different expression in lung cells. Nox1 is expressed in alveolar epithelial cells and vascular endothelial cells (Carnesecchi et al., 2009;
Takemura et al., 2010). In contrast, Nox2 is expressed in inflammatory cells, particularly in macrophages (Soucy-Faulkner et al., 2010).

$\mathrm{XO}$ is another ROS-producing enzyme involved in the lung injuries after IAV infection (Akaike et al., 1990; Chida et al., 2018). Expression of XO was shown to increase in the lung after IAV infection in response to pro-inflammatory cytokines (Pfeffer et al., 1994). Allopurinol, an inhibitor of XO, reduced mortality of mice infected with IAV/Kumamoto and IAV/PR8 (Akaike et al., 1990; Chida et al., 2018). These results suggest that $X O$ could be another target molecule for anti-influenza agents.

$\mathrm{NO}$ also contributes to the pathogenesis of IAV infections by being converted to a highly reactive radical peroxynitrite through reaction with superoxide (Peterhans, 1997). Expression of iNOS in lung epithelial cells and macrophages is enhanced by pro-inflammatory cytokines (Asano et al., 1994). Treatment with the NOS inhibitor, $N^{\omega}{ }_{-}$ monomethyl-L-arginine, successfully reduced the mortality of mice infected with IAV/Kumamoto (Akaike et al., 1996), suggesting that NOS could be also a plausible target molecule for anti-influenza agents.

\section{$\mathrm{PrPC}^{\mathrm{C}}$ and its anti-oxidative function}

$\mathrm{PrPC}^{\mathrm{C}}$ is a membrane glycoprotein tethered to the outer cell membrane via a glycosylphosphatidylinositol (GPI) anchor moiety and is expressed most abundantly in brains, particularly by neurons, and to a lesser extent in non-neuronal tissues including hearts, kidneys, and lungs (Prusiner, 1998). Conformational conversion of PrPC into the abnormally folded, amyloidogenic isoform of PrP, PrPSc, which leads to accumulation of PrPSc in the brain, is a key pathogenic event in prion diseases, a group of neurodegenerative disorders, which include Creutzfeldt-Jakob disease in humans and scrapie and bovine spongiform encephalopathy in animals (Prusiner, 1998). Indeed, we and others have shown that mice devoid of $\operatorname{PrPC}^{\mathrm{C}}\left(\operatorname{Prnp}^{0 / 0}\right)$ are resistant to prions, the causative agents of prion diseases (Bueler et al., 1993; Manson et al., 1994; Prusiner et al., 1993; Sakaguchi et al., 1995). Prnp $0 / 0$ mice neither produced PrPSc, or prions, in their brains nor developed the disease even after intracerebral inoculation with prions (Bueler et al., 1993; Manson et al., 1994; Prusiner et al., 1993; Sakaguchi et al., 1995). However, the molecular pathogenesis underlying the neurodegeneration remains largely unknown. 
Mouse $\mathrm{PrPC}$ is first translated as a precursor protein consisting of 254 amino acids, with the N-terminal 22 and C-terminal 23 hydrophobic amino acids being a signal peptide and a GPI-anchor signal sequence, respectively, which are removed on the biosynthesis of mature $\mathrm{PrP}^{\mathrm{C}}$ expressing on the cell surface (Prusiner, 1991) (Figure 2A). PrPC mainly consists of two domains, the $\mathrm{N}$ - and $\mathrm{C}$-terminal domains. The $\mathrm{N}$-terminal domain is highly flexible and lacks identifiable secondary structure (Donne et al., 1997). This part includes the so-called octapeptide repeat (OR) region, which is comprised of 5 tandem repeats of 8 amino acids (Jackson et al., 2001) (Figure 2A). The C-terminal domain forms a globular structure with three $\alpha$-helices and two short antiparallel $\beta$-strands (Riek et al., 1996) (Figure 2A). This part contains two N-type glycosylation sites and one disulfide bond between the second and the third helices (Prusiner, 1991) (Figure 2A). The OR region is very specific to PrPs and is considered to bind to $\mathrm{Cu}$ ions via histidine residues (Brown et al., 1997) (Figure 2A). It has been suggested that $\mathrm{PrPC}$ might exert an antioxidative function through regulating anti-oxidative enzymes, such as SOD1, via transfer of the bound $\mathrm{Cu}$ ions to the enzymes (Haigh and Brown, 2006). Indeed, Prnp ${ }^{0 / 0}$ mice have been reported to be vulnerable to ischemic brain injury, with enhanced oxidative stress in the injured brains (McLennan et al., 2004; Sakurai-Yamashita et al., 2005; Weise et al., 2004). PrP lacking the OR region failed to rescue $\mathrm{Prnp}^{0 / 0}$ mice from ischemic brain injury (Mitteregger et al., 2007). It was also reported that the hearts and kidneys of $\mathrm{Prnp}^{0 / 0}$ mice were vulnerable to ischemic injury with high oxidative stress (Zanetti et al., 2014; Zhang et al., 2015). Many other cellular functions have been also suggested for $\mathrm{PrPC}$, which include cell trafficking, adhesion, differentiation, signaling, and survival (Aguzzi et al., 2008).

\section{Protective role of $\mathrm{PrPC}$ against IAV infection}

We identified that Prnpo/0 mice were highly susceptible to intranasal infection with IAV/PR8, A/ Aichi/2/68 (H3N2), and A/WSN/33 (H1N1), with markedly elevated mortality, compared to control WT mice (Chida et al., 2018). Infected Prnpo/0 lungs were severely damaged, with higher infiltration of inflammatory cells and higher levels of inflammatory cytokines than control WT lungs. Virus titers were only slightly higher in infected Prnpo/0 lungs than in control WT lungs. PrPC was expressed by AT1 and AT2 cells and bronchiolar Clara epithelial cells in WT lungs (Chida et al., 2018). Higher apoptosis of AT2 and Clara cells was detected in infected Prnp ${ }^{0 / 0}$ lungs than in control WT lungs (Chida et al., 2018). In contrast, AT1 cells were not damaged in infected Prnp $^{0 / 0}$ and WT lungs (Chida et al., 2018), consistent with IAV/PR8 infection not damaging AT1 cells in C57BL/6 mice (Yamada et al., 2012). ROS levels were also higher in infected $\mathrm{Prnp}^{0 / 0}$ lungs compared to control WT lungs (Chida et al., 2018). Treatment with butylated hydroxyanisole, a ROS scavenger, decreased the mortality of infected Prnp $0 / 0$ mice to that of control WT mice (Chida et al., 2018). We also showed that the ROS-producing enzyme XO was higher in Prnpo/0 lungs than in WT lungs after infection with IAV/PR8, and treatment with the $\mathrm{XO}$ inhibitor allopurinol reduced the mortality of $P r n p^{0 / 0}$ and WT mice to a similar rate (Chida et al., 2018). These results suggest that PrPC could play an anti-oxidative role to reduce ROS levels in IAV-infected lungs, thereby providing some protection against lethal infection with IAVs, and that the higher expression of $X O$ leading to production of higher levels of ROS in IAV-infected Prnp ${ }^{0 / 0}$ lungs could be attributable to the higher mortality of IAVinfected Prnp ${ }^{0 / 0}$ mice (Figure 2B).

Mouse PrP with a deletion of the $\mathrm{Cu}$ ion-binding OR region failed to rescue $P_{r n p} / 0$ mice from lethal infection with IAV/PR8 (Chida et al., 2018). Cu ion content and SOD1 activity were lower in infected Prnpo/0 lungs than in control WT mice (Chida et al., 2018). $\operatorname{Tg}(\operatorname{PrP} \Delta \mathrm{OR}) / \mathrm{Prnp}^{0 / 0}$ mice transgenically expressing mouse $\operatorname{PrP}$ with a deletion of the OR region on the $\operatorname{Prnp}^{0 / 0}$ background also showed lower $\mathrm{Cu}$ ion content, lower SOD1 activity, and higher ROS levels in their lungs after infection with IAVI PR8 (Chida et al., 2018). These results suggest that the OR region plays an important role for $\mathrm{PrPC}^{\mathrm{C}}$ to protect against IAV infection probably through regulation of the $\mathrm{Cu}$ ion content and SOD1 activity in IAV-infected lungs (Figure 2B). IAVs primarily infect lung epithelial cells and then cause oxidative stress in them (Liu et al., 2017). It is thus possible that $\mathrm{Prnp}^{0 / 0}$ epithelial cells could not sufficiently combat the oxidative stress due to lack of $\mathrm{PrPC}^{\mathrm{C}}$, therefore undergoing apoptosis more easily than WT epithelial cells after IAV infection, provoking higher inflammatory responses leading to higher production of inflammatory cytokines in infected Prnp $0 / 0$ lungs, eventually causing higher mortality of $P r n p^{0 / 0}$ mice after infection with IAVs. PrPC has been also reported to exert a protective function against other virus infections, including encephalomyocarditis virus $B$ variant, herpes simplex virus type 1, human immunodeficiency virus type 1 , coxsackievirus B3, adenovirus 5 , and poliovirus-1 (Sakaguchi and Chida, 2018). However, whether the 
A

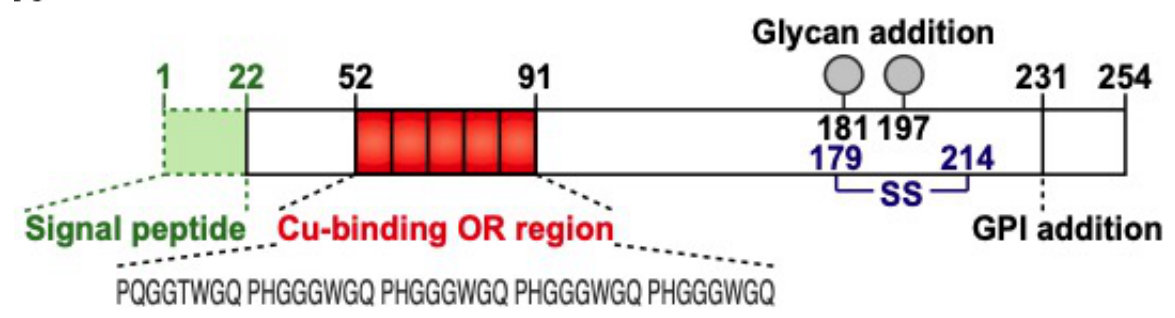

B

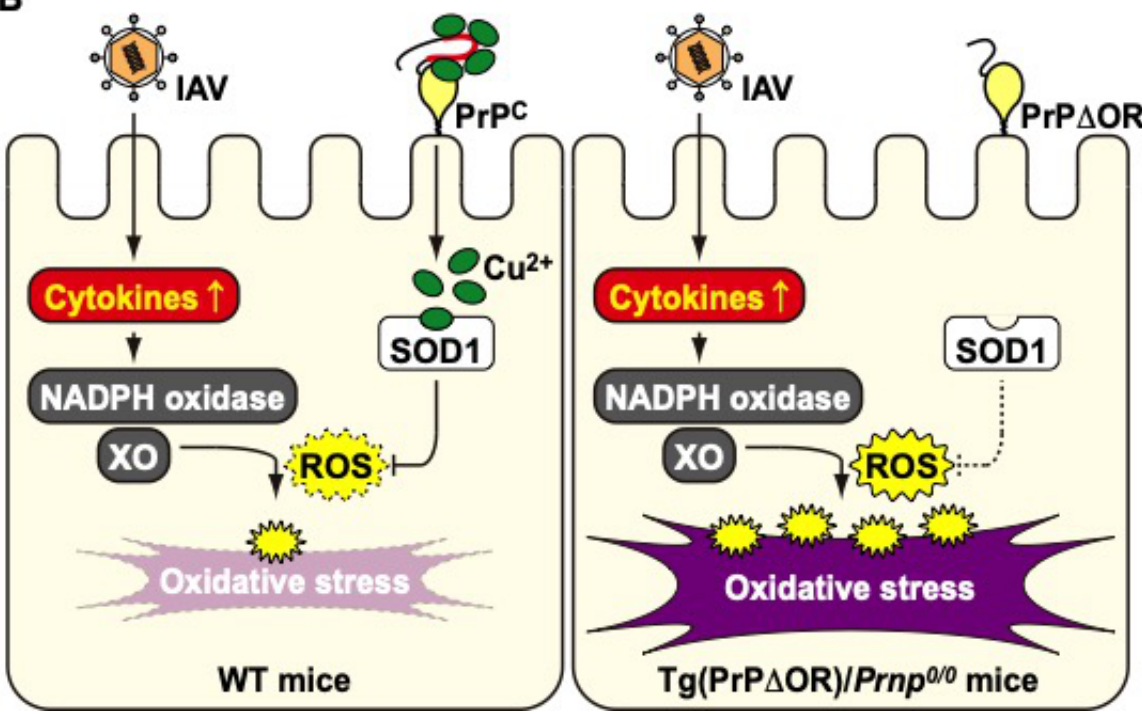

Figure 2. Anti-oxidative role of PrPC against IAV infection. (A) Schematic diagram of PrPC. SS: disulfide bound. (B) IAV infection induces production of inflammatory cytokines leading to elevated expression of NADPH oxidase and XO, eventually resulting in production of ROS causing oxidative stress. $\mathrm{PrPC}^{\mathrm{C}}$ functions to reduce ROS through regulation of SOD1 by transferring the $\mathrm{Cu}$ ions that are bound to the OR region. However, PrP $\triangle O R$ has no antioxidative activity since it cannot bind to $\mathrm{Cu}$ ions due to lack of the $\mathrm{OR}$ region, causing higher production of ROS and eventually higher oxidative stress.

anti-oxidative function of $\mathrm{PrPC}$ is relevant to its protective role against these virus infections remains to be determined.

\section{Conclusions}

It has been demonstrated that inhibition of the ROSgenerating enzymes or administration of antioxidant enzymes successfully protected mice from lethal infection with IAVs (Akaike et al., 1990; Oda et al., 1989; Vlahos and Selemidis, 2014), suggesting that anti-oxidative therapies targeting cellular oxidative molecules could be beneficial against IAV infections. The currently available agents such as NA inhibitors target viral molecules, thereby stimulating the emergence of drug-resistant IAVs. However, anti-influenza agents targeting host molecules are considered to greatly reduce the risk of emergence of drug-resistant IAVs or not to induce drug-resistant IAVs at all. Therefore, cellular antioxidative molecules involved in protection against IAV infection would be plausible targets for development of anti-influenza agents.

Our current findings suggest that $\mathrm{PrPC}^{\mathrm{C}}$ could be a novel target molecule for development of antioxidative therapeutics against IAV infection (Chida et al., 2018). It has been reported that $\operatorname{PrPC}^{\mathrm{C}}$ protected neurons from anisomycin-induced apoptosis via interaction with stress-inducible protein 1 (STI1), a STI1-derived peptide, or anti-PrP antibodies (Chiarini et al., 2002; Zanata et al., 2002). Furthermore, the interaction with STI1 could 
be involved in $\mathrm{PrP}^{\mathrm{C}}$-dependent activation of $\mathrm{SOD}$ (Sakudo et al., 2005). It is thus interesting to investigate whether these ligands for $\mathrm{PrPC}$ could elicit anti-oxidative activity in lungs and protect against IAV infection. This is currently underway in our laboratory.

\section{Acknowledgements}

This study was partly supported to SS by JSPS KAKENHI Grant Number 19H03548, 17K19661, and $15 \mathrm{~K} 15380$, and Grant-in-Aid for Scientific Research on Innovative Areas (Brain Protein Aging and Dementia Control) Grant Number 17H05701 from MEXT, and to JC by JSPS KAKENHI Grant Number $19 K 0893000$ and 16K10029.

\section{Disclosure Statement}

No competing financial interests exist.

\section{References}

Aguzzi, A., Baumann, F., and Bremer, J. (2008). The prion's elusive reason for being. Annu. Rev. Neurosci. 31, 439-477.

Akaike, T. (2001). Role of free radicals in viral pathogenesis and mutation. Rev. Med. Virol. 11, 87-101.

Akaike, T., Ando, M., Oda, T., Doi, T., ljiri, S., Araki, S., and Maeda, H. (1990). Dependence on O2generation by xanthine oxidase of pathogenesis of influenza virus infection in mice. J. Clin. Invest 85, 739-745. 10.1172/JCl114499

Akaike, T., Noguchi, Y., ljiri, S., Setoguchi, K., Suga, M., Zheng, Y.M., Dietzschold, B., and Maeda, H. (1996). Pathogenesis of influenza virus-induced pneumonia: involvement of both nitric oxide and oxygen radicals. Proc. Natl. Acad. Sci. USA 93, 2448-2453.

Antosova, M., Mokra, D., Pepucha, L., Plevkova, J., Buday, T., Sterusky, M., and Bencova, A. (2017). Physiology of nitric oxide in the respiratory system. Physiol. Res. 66, S159-S172.

Asano, K., Chee, C.B., Gaston, B., Lilly, C.M., Gerard, C., Drazen, J.M., and Stamler, J.S. (1994). Constitutive and inducible nitric oxide synthase gene expression, regulation, and activity in human lung epithelial cells. Proc. Natl. Acad. Sci. USA 91, 10089-10093. 10.1073/pnas. 91.21.10089

Brown, D.R., Qin, K., Herms, J.W., Madlung, A., Manson, J., Strome, R., Fraser, P.E., Kruck, T., von Bohlen, A., Schulz-Schaeffer, W., et al. (1997). The cellular prion protein binds copper in vivo. Nature 390, 684-687.

Bueler, H., Aguzzi, A., Sailer, A., Greiner, R.A., Autenried, P., Aguet, M., and Weissmann, C.
(1993). Mice devoid of PrP are resistant to scrapie. Cell 73, 1339-1347.

Carnesecchi, S., Deffert, C., Pagano, A., GarridoUrbani, S., Metrailler-Ruchonnet, I., Schappi, M., Donati, Y., Matthay, M.A., Krause, K.H., and Barazzone Argiroffo, C. (2009). NADPH oxidase-1 plays a crucial role in hyperoxia-induced acute lung injury in mice. Am. J. Respir. Crit. Care Med. 180, 972-981. 10.1164/rccm.200902-0296OC

Chiarini, L.B., Freitas, A.R., Zanata, S.M., Brentani, R.R., Martins, V.R., and Linden, R. (2002). Cellular prion protein transduces neuroprotective signals. Embo J. 21, 3317-3326.

Chida, J., Hara, H., Yano, M., Uchiyama, K., Das, N.R., Takahashi, E., Miyata, H., Tomioka, Y., Ito, T., Kido, H., et al. (2018). Prion protein protects mice from lethal infection with influenza $A$ viruses. PLoS Pathog. 14, e1007049. 10.1371/ journal.ppat.1007049

Chutinimitkul, S., Herfst, S., Steel, J., Lowen, A.C., Ye, J., van Riel, D., Schrauwen, E.J., Bestebroer, T.M., Koel, B., Burke, D.F., et al. (2010). Virulence-associated substitution D222G in the hemagglutinin of 2009 pandemic influenza $\mathrm{A}(\mathrm{H} 1 \mathrm{~N} 1)$ virus affects receptor binding. J. Virol. 84, 11802-11813. 10.1128/JVI.01136-10

Donne, D.G., Viles, J.H., Groth, D., Mehlhorn, I., James, T.L., Cohen, F.E., Prusiner, S.B., Wright, P.E., and Dyson, H.J. (1997). Structure of the recombinant full-length hamster prion protein $\operatorname{PrP}(29-231)$ : the $N$ terminus is highly flexible. Proc. Natl. Acad. Sci. USA 94, 13452-13457.

Fiore, A.E., Shay, D.K., Broder, K., Iskander, J.K., Uyeki, T.M., Mootrey, G., Bresee, J.S., and Cox, N.S. (2008). Prevention and control of influenza: recommendations of the Advisory Committee on Immunization Practices (ACIP), 2008. MMWR Recommendations and reports : Morbidity and mortality weekly report Recommendations and reports/Centers for Disease Control 57, 1-60.

Folz, R.J., Abushamaa, A.M., and Suliman, H.B. (1999). Extracellular superoxide dismutase in the airways of transgenic mice reduces inflammation and attenuates lung toxicity following hyperoxia. J. Clin. Invest. 103, 1055-1066. 10.1172/JCI3816

Folz, R.J., Guan, J., Seldin, M.F., Oury, T.D., Enghild, J.J., and Crapo, J.D. (1997). Mouse extracellular superoxide dismutase: primary structure, tissue-specific gene expression, chromosomal localization, and lung in situ hybridization. Am. J. Respir. Cell. Mol. Biol. 17, 393-403. 10.1165/ajrcmb.17.4.2826

Guarda, G., Zenger, M., Yazdi, A.S., Schroder, K., Ferrero, I., Menu, P., Tardivel, A., Mattmann, C., and Tschopp, J. (2011). Differential expression of 
NLRP3 among hematopoietic cells. J. Immunol. 186, 2529-2534. 10.4049/jimmunol.1002720

Haigh, C.L., and Brown, D.R. (2006). Prion protein reduces both oxidative and non-oxidative copper toxicity. J. Neurochem. 98, 677-689.

Hofstetter, A.R., De La Cruz, J.A., Cao, W., Patel, J., Belser, J.A., McCoy, J., Liepkalns, J.S., Amoah, S., Cheng, G., Ranjan, P., et al. (2016). NADPH Oxidase 1 Is Associated with Altered Host Survival and T Cell Phenotypes after Influenza A Virus Infection in Mice. PLoS One 11, e0149864. 10.1371/journal.pone. 0149864

Imai, Y., Kuba, K., Neely, G.G., Yaghubian-Malhami, R., Perkmann, T., van Loo, G., Ermolaeva, M.,

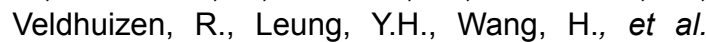
(2008). Identification of oxidative stress and Tolllike receptor 4 signaling as a key pathway of acute lung injury. Cell 133, 235-249. 10.1016/ j.cell.2008.02.043

Iwasaki, A., and Pillai, P.S. (2014). Innate immunity to influenza virus infection. Nat. Rev. Immunol. 14, 315-328. 10.1038/nri3665

Jackson, G.S., Murray, I., Hosszu, L.L., Gibbs, N., Waltho, J.P., Clarke, A.R., and Collinge, J. (2001). Location and properties of metal-binding sites on the human prion protein. Proc. Natl. Acad. Sci. USA 98, 8531-8535.

Kinnula, V.L., and Crapo, J.D. (2003). Superoxide dismutases in the lung and human lung diseases. Am. J. Respir. Crit. Care Med. 167, 1600-1619. 10.1164/rccm.200212-1479SO

Kinnula, V.L., Crapo, J.D., and Raivio, K.O. (1995). Generation and disposal of reactive oxygen metabolites in the lung. Lab. Invest. 73, 3-19.

La Gruta, N.L., Kedzierska, K., Stambas, J., and Doherty, P.C. (2007). A question of selfpreservation: immunopathology in influenza virus infection. Immunol. Cell. Biol. 85, 85-92. 10.1038/ sj.icb. 7100026

Liu, M., Chen, F., Liu, T., Chen, F., Liu, S., and Yang, J. (2017). The role of oxidative stress in influenza virus infection. Microbes Infect. 19, 580-586. 10.1016/j.micinf.2017.08.008

Manson, J.C., Clarke, A.R., McBride, P.A., McConnell, I., and Hope, J. (1994). PrP gene dosage determines the timing but not the final intensity or distribution of lesions in scrapie pathology. Neurodegeneration 3, 331-340.

Marques, M., Ramos, B., Soares, A.R., and Ribeiro, D. (2019). Cellular Proteostasis During Influenza A Virus Infection-Friend or Foe? Cells 810.3390/ cells8030228

McLennan, N.F., Brennan, P.M., McNeill, A., Davies, I., Fotheringham, A., Rennison, K.A., Ritchie, D., Brannan, F., Head, M.W., Ironside, J.W., et al.
(2004). Prion protein accumulation and neuroprotection in hypoxic brain damage. Am. J. Pathol. 165, 227-235.

Mitteregger, G., Vosko, M., Krebs, B., Xiang, W., Kohlmannsperger, V., Nolting, S., Hamann, G.F., and Kretzschmar, H.A. (2007). The role of the octarepeat region in neuroprotective function of the cellular prion protein. Brain Pathol. 17, 174-183.

Nickol, M.E., and Kindrachuk, J. (2019). A year of terror and a century of reflection: perspectives on the great influenza pandemic of 1918-1919. BMC Infect. Dis. 19, 117. 10.1186/s12879-019-3750-8

Nunez, I.A., and Ross, T.M. (2019). A review of $\mathrm{H} 5 \mathrm{Nx}$ avian influenza viruses. Ther. Adv. Vaccines Immunother. 7, 2515135518821625 . 10.1177/2515135518821625

Oda, T., Akaike, T., Hamamoto, T., Suzuki, F., Hirano, T., and Maeda, H. (1989). Oxygen radicals in influenza-induced pathogenesis and treatment with pyran polymer-conjugated SOD. Science 244, 974-976.

Pechkovsky, D.V., Zissel, G., Goldmann, T., Einhaus, M., Taube, C., Magnussen, H., Schlaak, M., and Muller-Quernheim, J. (2002). Pattern of NOS2 and NOS3 mRNA expression in human A549 cells and primary cultured AEC II. Am. J. Physiol. Lung Cell. Mol. Physiol. 282, L684-692. 10.1152/ajplung.00320.2000

Peterhans, E. (1997). Reactive oxygen species and nitric oxide in viral diseases. Biol. Trace Elem. Res. 56, 107-116. 10.1007/BF02778986

Pfeffer, K.D., Huecksteadt, T.P., and Hoidal, J.R. (1994). Xanthine dehydrogenase and xanthine oxidase activity and gene expression in renal epithelial cells. Cytokine and steroid regulation. J. Immunol. 153, 1789-1797.

Pietarinen-Runtti, P., Lakari, E., Raivio, K.O., and Kinnula, V.L. (2000). Expression of antioxidant enzymes in human inflammatory cells. Am. J. Physiol. Cell. Physiol. 278, C118-125. 10.1152/ ajpcell.2000.278.1.C118

Pinto, L.H., and Lamb, R.A. (2006). The M2 proton channels of influenza $A$ and $B$ viruses. J. Biol. Chem. 281, 8997-9000. 10.1074/jbc.R500020200

Prusiner, S.B. (1991). Molecular biology of prion diseases. Science 252, 1515-1522.

Prusiner, S.B. (1998). Prions. Proc. Natl. Acad. Sci. USA 95, 13363-13383.

Prusiner, S.B., Groth, D., Serban, A., Koehler, R., Foster, D., Torchia, M., Burton, D., Yang, S.L., and DeArmond, S.J. (1993). Ablation of the prion protein (PrP) gene in mice prevents scrapie and facilitates production of anti-PrP antibodies. Proc. Natl. Acad. Sci. USA 90, 10608-10612. 
Ricciardolo, F.L., Sterk, P.J., Gaston, B., and Folkerts, G. (2004). Nitric oxide in health and disease of the respiratory system. Physiol. Rev. 84, 731-765. 10.1152/physrev.00034.2003

Riek, R., Hornemann, S., Wider, G., Billeter, M., Glockshuber, R., and Wuthrich, K. (1996). NMR structure of the mouse prion protein domain $\operatorname{PrP}(121-321)$. Nature 382, 180-182.

Robson, C., Baskar, S.R., Booy, R., Ferguson, P.E., Gilroy, N., Kok, J., Sandaradura, I., and Dwyer, D. (2019). Influenza: overview on prevention and therapy. Aust. Prescr. 42, 51-55. 10.18773/ austprescr.2019.013

Rossman, J.S., and Lamb, R.A. (2011). Influenza virus assembly and budding. Virology 411, 229-236. 10.1016/j.virol.2010.12.003

Sakaguchi, S., and Chida, J. (2018). Roles of Prion Protein in Virus Infections. DNA Cell Biol. 37, 808-811. 10.1089/dna.2018.4402

Sakaguchi, S., Katamine, S., Shigematsu, K., Nakatani, A., Moriuchi, R., Nishida, N., Kurokawa, K., Nakaoke, R., Sato, H., Jishage, K., et al. (1995). Accumulation of proteinase K-resistant prion protein $(\mathrm{PrP})$ is restricted by the expression level of normal PrP in mice inoculated with a mouse-adapted strain of the Creutzfeldt-Jakob disease agent. J. Virol. 69, 7586-7592.

Sakudo, A., Lee, D.C., Li, S., Nakamura, T., Matsumoto, Y., Saeki, K., Itohara, S., Ikuta, K., and Onodera, T. (2005). PrP cooperates with STI1 to regulate SOD activity in PrP-deficient neuronal cell line. Biochem. Biophys. Res. Commun. 328, 14-19.

Sakurai-Yamashita, Y., Sakaguchi, S., Yoshikawa, D., Okimura, N., Masuda, Y., Katamine, S., and Niwa, M. (2005). Female-specific neuroprotection against transient brain ischemia observed in mice devoid of prion protein is abolished by ectopic expression of prion protein-like protein. Neuroscience 136, 281-287.

Selemidis, S., Seow, H.J., Broughton, B.R., Vinh, A., Bozinovski, S., Sobey, C.G., Drummond, G.R., and Vlahos, R. (2013). Nox1 oxidase suppresses influenza a virus-induced lung inflammation and oxidative stress. PLoS One 8, e60792. 10.1371/ journal.pone.0060792

Sgarbanti, R., Amatore, D., Celestino, I., Marcocci, M.E., Fraternale, A., Ciriolo, M.R., Magnani, M., Saladino, R., Garaci, E., Palamara, A.T., et al. (2014). Intracellular redox state as target for antiinfluenza therapy: are antioxidants always effective? Curr. Top. Med. Chem. 14, 2529-2541.

Shinya, K., Ebina, M., Yamada, S., Ono, M., Kasai, N., and Kawaoka, Y. (2006). Avian flu: influenza virus receptors in the human airway. Nature 440 , 435-436. 10.1038/440435a

Sidwell, R.W., Huffman, J.H., Bailey, K.W., Wong, M.H., Nimrod, A., and Panet, A. (1996). Inhibitory effects of recombinant manganese superoxide dismutase on influenza virus infections in mice. Antimicrob. Agents Chemother. 40, 2626-2631.

Snelgrove, R.J., Edwards, L., Rae, A.J., and Hussell, T. (2006). An absence of reactive oxygen species improves the resolution of lung influenza infection. Eur. J. Immunol. 36, 1364-1373. 10.1002/eji.200635977

Soucy-Faulkner, A., Mukawera, E., Fink, K., Martel, A., Jouan, L., Nzengue, Y., Lamarre, D., Vande Velde, C., and Grandvaux, N. (2010). Requirement of NOX2 and reactive oxygen species for efficient RIG-I-mediated antiviral response through regulation of MAVS expression. PLoS Pathog. 6, e1000930. 10.1371/journal.ppat. 1000930

Su, W.Y., Folz, R., Chen, J.S., Crapo, J.D., and Chang, L.Y. (1997). Extracellular superoxide dismutase mRNA expressions in the human lung by in situ hybridization. Am. J. Respir. Cell. Mol. Biol. 16, 162-170. 10.1165/ajrcmb.16.2.9032123

Suliman, H.B., Ryan, L.K., Bishop, L., and Folz, R.J. (2001). Prevention of influenza-induced lung injury in mice overexpressing extracellular superoxide dismutase. Am. J. Physiol. Lung Cell. Mol. Physiol. 280, L69-78. 10.1152/ajplung. 2001.280.1.L69

Takemura, Y., Goodson, P., Bao, H.F., Jain, L., and Helms, M.N. (2010). Rac1-mediated NADPH oxidase release of $\mathrm{O} 2$ - regulates epithelial sodium channel activity in the alveolar epithelium. Am. J. Physiol. Lung Cell. Mol. Physiol. 298, L509-520. 10.1152/ajplung.00230.2009

Thomas, P.G., Dash, P., Aldridge, J.R., Jr., Ellebedy, A.H., Reynolds, C., Funk, A.J., Martin, W.J., Lamkanfi, M., Webby, R.J., Boyd, K.L., et al. (2009). The intracellular sensor NLRP3 mediates key innate and healing responses to influenza $A$ virus via the regulation of caspase- 1 . Immunity 30, 566-575. 10.1016/j.immuni.2009.02.006

Tumpey, T.M., Basler, C.F., Aguilar, P.V., Zeng, H., Solorzano, A., Swayne, D.E., Cox, N.J., Katz, J.M., Taubenberger, J.K., Palese, P., et al. (2005). Characterization of the reconstructed 1918 Spanish influenza pandemic virus. Science 310, 77-80. 10.1126/science.1119392

van Riel, D., den Bakker, M.A., Leijten, L.M., Chutinimitkul, S., Munster, V.J., de Wit, E., Rimmelzwaan, G.F., Fouchier, R.A., Osterhaus, A.D., and Kuiken, T. (2010). Seasonal and pandemic human influenza viruses attach better 
to human upper respiratory tract epithelium than avian influenza viruses. Am. J. Pathol. 176, 1614-1618. 10.2353/ajpath.2010.090949

Vlahos, R., and Selemidis, S. (2014). NADPH oxidases as novel pharmacologic targets against influenza A virus infection. Mol. Pharmacol. 86, 747-759. 10.1124/mol.114.095216

Vlahos, R., Stambas, J., Bozinovski, S., Broughton, B.R., Drummond, G.R., and Selemidis, S. (2011). Inhibition of Nox2 oxidase activity ameliorates influenza A virus-induced lung inflammation. PLoS Pathog. 7, e1001271. 10.1371/journal.ppat. 1001271

Warner, R.L., Paine, R., 3rd, Christensen, P.J., Marletta, M.A., Richards, M.K., Wilcoxen, S.E., and Ward, P.A. (1995). Lung sources and cytokine requirements for in vivo expression of inducible nitric oxide synthase. Am. J. Respir. Cell. Mol. Biol. 12, 649-661. 10.1165/ajrcmb.12.6.7539274

Weise, J., Crome, O., Sandau, R., Schulz-Schaeffer, W., Bahr, M., and Zerr, I. (2004). Upregulation of cellular prion protein (PrPc) after focal cerebral ischemia and influence of lesion severity. Neurosci. Lett. 372, 146-150.

Yamada, Y., Limmon, G.V., Zheng, D., Li, N., Li, L., Yin, L., Chow, V.T., Chen, J., and Engelward, B.P.
(2012). Major shifts in the spatio-temporal distribution of lung antioxidant enzymes during influenza pneumonia. PLoS ONE 7, e31494. 10.1371/journal.pone.0031494

Yamayoshi, S., and Kawaoka, Y. (2019). Current and future influenza vaccines. Nat Med 25, 212-220. 10.1038/s41591-018-0340-z

Zanata, S.M., Lopes, M.H., Mercadante, A.F., Hajj, G.N., Chiarini, L.B., Nomizo, R., Freitas, A.R., Cabral, A.L., Lee, K.S., Juliano, M.A., et al. (2002). Stress-inducible protein 1 is a cell surface ligand for cellular prion that triggers neuroprotection. Embo J. 21, 3307-3316.

Zanetti, F., Carpi, A., Menabo, R., Giorgio, M., Schulz, R., Valen, G., Baysa, A., Massimino, M.L., Sorgato, M.C., Bertoli, A., et al. (2014). The cellular prion protein counteracts cardiac oxidative stress. Cardiovas. Res. 104, 93-102. 10.1093/cvr/ cvu194

Zhang, B., Cowden, D., Zhang, F., Yuan, J., Siedlak, S., Abouelsaad, M., Zeng, L., Zhou, X., O'Toole, J., Das, A.S., et al. (2015). Prion Protein Protects against Renal Ischemia/Reperfusion Injury. PLoS ONE 10, e0136923. 10.1371/journal.pone. 0136923 
